\title{
Feedback by massive stars and the emergence of superbubbles II. X-ray properties (Corrigendum)
}

\author{
Martin Krause $^{1,2}$, Roland Diehl ${ }^{1,2}$, Hans Böhringer ${ }^{1,2}$, Michael Freyberg ${ }^{1}$, and Daniel Lubos ${ }^{1}$ \\ ${ }^{1}$ Max-Planck-Institut für extraterrestrische Physik, Giessenbachstr. 1, 85741 Garching, Germany \\ e-mail: krause@mpe.mpg. de \\ ${ }^{2}$ Excellence Cluster Universe, Technische Universität München, Boltzmannstrasse 2, 85748 Garching, Germany
}

A\&A 566, A94 (2014), DOI: 10.1051/0004-6361/201423871

Key words. ISM: bubbles - ISM: structure - galaxies: ISM - hydrodynamics - instabilities - errata, addenda

\section{Corrected extent of the mixing region}

In Fig. 2 of Krause et al. (2014), we showed the density in a simulated superbubble as a function of radius averaged over the solid angle as seen from the geometrical centre of the main bubble. This plot was misleading as it implied an extent of the mixing region - responsible for much of the X-ray emission discussed in this paper - twice as large as it actually is. The reason is that due to the averaging over the solid angle, side cavities from other massive stars influence the plot. The true extent of the mixing region just before the first supernova explosion is $10 \mathrm{pc}$, and it extends from $X=40 \mathrm{pc}$ to $X=50 \mathrm{pc}$ as shown in Fig. 1. The contact surface is thus still resolved. The results and conclusions of the article are not affected by this, because the synthetic emission is derived from the numerical grid directly.

\section{References}

Krause, M., Diehl, R., Böhringer, H., Freyberg, M., \& Lubos, D. 2014, A\&A, 566, A94

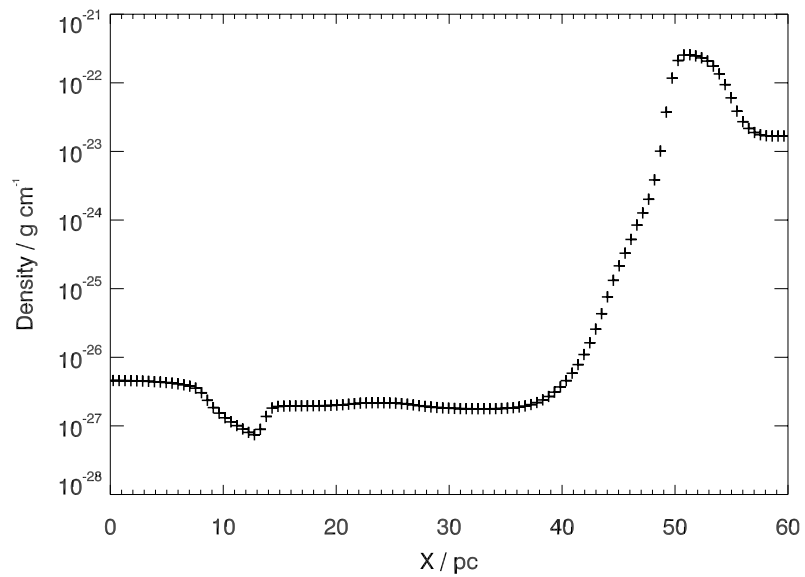

Fig. 1. This figure replaces Fig. 2 of the original publication. It shows the run of the density on a linear slice along the positive $X$-axis for run 3S1-hr at time $4.598 \mathrm{Myr}$, i.e. $2139 \mathrm{yr}$ before the central most massive star (at $X=0$ ) explodes. Each "+"-Symbol shows the value of the density in one grid cell. The mixing region is resolved with about 20 cells, and extends from 40 to $50 \mathrm{pc}$. 